\title{
Consent procedures and relationship with outcome in the Rapid Intervention with Glyceryl trinitrate in Hypertensive stroke Trial-2 (RIGHT-2)
}

Polly Scutt, Mark Dixon, Jason P Appleton, Harriet Howard, Diane Havard, Lisa J

Woodhouse, Philip M Bath; for RIGHT-2 investigators

Stroke, Division of Clinical Neuroscience, University of Nottingham, Nottingham UK

\section{Background}

Obtaining consent in emergency situations is challenging. Proxy consent allows patients to be recruited when they lack capacity, a common scenario in stroke patients. The rapid intervention with glyceryl trinitrate in hypertensive stroke trial-2 (RIGHT-2) recruits patients in the pre-hospital setting within 4 hours of stroke onset.

\section{Methods}

In RIGHT-2, informed or proxy consent is taken in the ambulance. A brief assessment of capacity is performed by the paramedic. Patients with capacity provide consent and in patients without capacity, proxy consent is obtained from a relative, carer or friend, or by the paramedic, witnessed by a crew member.

\section{Results}

Of 879 participants enrolled into RIGHT-2 as of $15^{\text {th }}$ December $2017,468(53.2 \%)$ participants gave their own consent; proxy consent was given by a relative/carer/friend for $325(37 \%)$ and by a paramedic for 85 (9.7\%). Participants who consented themselves were younger, had less dependency and had less severe strokes than those with proxy consent. Participants who gave their own consent had a lower rate of intracerebral haemorrhage ( $9 \%$ vs $16 \%)$ and a higher rate of non-stroke ( $20 \%$ vs $13 \%)$ as their final diagnosis than those who gave proxy consent. Consenting patients had better scores for dependency, cognition, disability and quality of life at day 90 than those recruited via proxy consent.

\section{Conclusion}

Proxy consent can ensure participants are enrolled rapidly into emergency clinical trials where they may otherwise be excluded due to lack of capacity. These patients have more severe strokes and therefore poorer clinical outcomes.

The RIGHT-2 trial is registered: ISRCTN26986053 